Bull. Austral. Math. Soc.

VOL. 52 (1995) [353-358]

\title{
TWO CHARACTERISATIONS OF A GAMMA MIXTURE DISTRIBUTION
}

\author{
M. Gharib
}

\begin{abstract}
Two characterisations are obtained for a gamma mixture distribution. The first is a generalisation of a result of Engel, Zijlstra and Philips [4] and the second is based on Gumbel's bivariate exponential distribution. The two characterisations are of direct relevance to some practical problems.
\end{abstract}

\section{INTRODUCTION}

Finite mixture distributions have recently received increasing attention in the statistical literature partly because of interest in their mathematical properties but mainly because they arise in an extremely wide variety of disciplines ranging from atomic physics to life testing, reliability and microbiology. Ashton [2] used a gamma mixture distribution in studying the distribution of time gaps in road traffic flow. In biology a common method (see Prentice [8]) for introducing extra-binomial variation in dilution analysis is to assume a mixture model. O'Neill [7] used a gamma mixture model for analysing the dilution data.

In the present paper two characterisations are obtained for a mixture of two gamma distributions. The first is an extension of the result of [4] concerning the characterisation of the gamma distribution by the negative binomial distribution which is based on the one-to-one map, generated by the Poisson process, between the continuous probability distributions on $[0, \infty)$ and the discrete distributions defined on $\{0,1,2, \ldots\}$. Our second result, which seems to be new in the literature, characterises the gamma mixture distribution using Gumbel's bivariate exponential distribution. The motivation here is the important role of the bivariate exponential distributions in representing the waiting time in two dimensional Poisson processes (see [6]).

\section{THE FIRST CHARACTERISATION}

Consider a homogeneous Poisson process with parameter $\lambda=1$ and let $N(Y)$ be the number of points in the time-interval $[0, Y)$ with $Y$ a non-negative random variable.

Received 16 January 1995

Copyright Clearance Centre, Inc. Serial-fee code: 0004-9729/95 \$A2.00+0.00. 
TheOREM. $Y$ has a gamma mixture distribution with respective parameters $\left(\alpha_{i}, \beta_{i}\right), i=1,2$ if and only if $N(Y)$ is distributed as a mixture of two negative binomial distributions with respective parameters $\left(\alpha_{i}, \beta_{i} /\left(1+\beta_{i}\right)\right), i=1,2$.

Proof: Suppose $Y$ has a gamma mixture distribution with density function

$$
\begin{gathered}
h(y)=\frac{\lambda_{1}}{\Gamma\left(\alpha_{1}\right)} \beta_{1}^{\alpha_{1}} y^{\alpha_{1}-1} e^{-\beta_{1} y}+\frac{\lambda_{2}}{\Gamma\left(\alpha_{2}\right)} \beta_{2}^{\alpha_{2}} y^{\alpha_{2}-1} e^{-\beta_{2} y}, \\
y>0 ; \alpha_{i}>0 ; \beta_{i}>0 ; i=1,2 ; 0 \leqslant \lambda_{1} \leqslant 1 ; \lambda_{1}+\lambda_{2}=1 .
\end{gathered}
$$

Then, we have

$$
\begin{aligned}
& P(N(Y)=k)=\int_{0}^{\infty} \frac{y^{k}}{k !} e^{-y} h(y) d y \\
& =\lambda_{1} \frac{\Gamma\left(k+\alpha_{1}\right)}{\Gamma\left(\alpha_{1}\right) k !}\left(\frac{\beta_{1}}{1+\beta_{1}}\right)^{\alpha_{1}}\left(\frac{1}{1+\beta_{1}}\right)^{k} \int_{0}^{\infty} \frac{\left(1+\beta_{1}\right)^{k+\alpha_{1}}}{\Gamma\left(k+\alpha_{1}\right)} y^{k+\alpha_{1}-1} e^{-\left(1+\beta_{1}\right) y} d y \\
& \quad+\lambda_{2} \frac{\Gamma\left(k+\alpha_{2}\right)}{\Gamma\left(\alpha_{2}\right) k !}\left(\frac{\beta_{2}}{1+\beta_{2}}\right)^{\alpha_{2}}\left(\frac{1}{1+\beta_{2}}\right)^{k} \int_{0}^{\infty} \frac{\left(1+\beta_{2}\right)^{k+\alpha_{2}}}{\Gamma\left(k+\alpha_{2}\right)} y^{k+\alpha_{2}-1} \mathrm{e}^{-\left(1+\beta_{2}\right) y} d y \\
& =\lambda_{1}\left(\begin{array}{c}
k+\alpha_{1}-1 \\
k
\end{array}\right)\left(\frac{\beta_{1}}{1+\beta_{1}}\right)^{\alpha_{1}}\left(1-\frac{\beta_{1}}{1+\beta_{1}}\right)^{k} \\
& \quad+\lambda_{2}\left(\begin{array}{c}
k+\alpha_{2}-1 \\
k
\end{array}\right)\left(\frac{\beta_{2}}{1+\beta_{2}}\right)^{\alpha_{2}}\left(1-\frac{\beta_{2}}{1+\beta_{2}}\right)^{k} \\
& k=0,1,2, \ldots
\end{aligned}
$$

Hence, the distribution of $N(Y)$ is a mixture of two negative binomial distributions with parameters $\left(\alpha_{i}, \beta_{i}\left(1+\beta_{i}\right)^{-1}\right), i=1,2$ respectively.

We now prove the "only if" part.

We have

$$
\int_{0}^{\infty} \frac{y^{k}}{k !} e^{-y} d H(y)=\sum_{i=1}^{2} \lambda_{i}\left(\begin{array}{c}
k+\alpha_{i}-1 \\
k
\end{array}\right)\left(\frac{\beta_{i}}{1+\beta_{i}}\right)^{\alpha_{i}}\left(\frac{1}{1+\beta_{i}}\right)^{k}, \quad k=0,1,2, \ldots
$$

where $H(y)$ is the distribution function of $Y$.

Now, let $g(s)$ be the Laplace-Stieltjes transform of the probability measure defined by $\boldsymbol{H}(y)$. Therefore

$$
g(s)=\int_{0}^{\infty} e^{-s y} d H(y) ; \quad \operatorname{Re}(s)>0 .
$$

Differentiating (2.3) $k$ times, we get

$$
g^{(k)}(s)=\int_{0}^{\infty}(-y)^{k} e^{-s y} d H(y)
$$


Using (2.4) we observe that (2.2) is equivalent to

$$
\frac{(-1)^{k}}{k !} g^{(k)}(1)=\sum_{i=1}^{2} \lambda_{i}\left(\begin{array}{c}
k+\alpha_{i}-1 \\
k
\end{array}\right)\left(\frac{\beta_{i}}{1+\beta_{i}}\right)^{\alpha_{i}}\left(\frac{1}{1+\beta_{i}}\right)^{k} ; \quad k=0,1,2, \ldots
$$

Multiplying both sides of $(2.5)$ by $(-s)^{k}$ and then summing for all values of $k$, we get

$$
\begin{aligned}
\sum_{0}^{\infty} \frac{g^{(k)}(1)}{k !} s^{k} & =\sum_{i=1}^{2} \lambda_{i}\left(\frac{\beta_{i}}{\left(1+\beta_{i}\right)}\right)^{\alpha_{i}} \sum_{k=0}^{\infty}\left(\begin{array}{c}
k+\alpha_{i}-1 \\
k
\end{array}\right)\left(\frac{-s}{1+\beta_{i}}\right)^{k} \\
& =\sum_{i=1}^{2} \lambda_{i}\left(\frac{\beta_{i}}{1+\beta_{i}}\right)^{\alpha_{i}}\left(1+\frac{s}{1+\beta_{i}}\right)^{-\alpha_{i}} \\
& =\sum_{i=1}^{2} \lambda_{i}\left(\frac{\beta_{i}}{s+1+\beta_{i}}\right)^{\alpha_{i}}
\end{aligned}
$$

Now, the left hand side of (2.6) is the Maclaurin expansion of the function $g(1+s)$. Hence,

$$
g(1+s)=\sum_{i=1}^{2} \lambda_{i}\left(\frac{\beta_{i}}{1+s+\beta_{i}}\right)^{\alpha_{i}}
$$

or,

$$
g(s)=\sum_{i=1}^{2} \lambda_{i}\left(\frac{\beta_{i}}{s+\beta_{i}}\right)^{\alpha_{i}}
$$

The right hand side of (2.7) is the Laplace transform of a mixture of two gamma distributions with respective parameters $\left(\alpha_{i}, \beta_{i}\right), i=1,2$. Since the Laplace transform is a one-to-one mapping, the proof is complete.

REMARKS.

1. In the special case $\alpha_{1}=\alpha_{2}=1$, that is, $Y$ is distributed as a mixture of two exponential distributions with respective parameters $\beta_{1}$ and $\beta_{2}, N(Y)$ has a mixture distribution of two geometric distributions with respective parameters $\beta_{1} /\left(1+\beta_{1}\right)$ and $\beta_{2} /\left(1+\beta_{2}\right)$.

2. The above theorem can be extended, easily, to the case of a mixture of any finite number of gamma distributions. 


\section{ThE SECOND ChaRACTERISATION}

Let $X=\left(X_{1}, X_{2}\right)$ be a vector of non-negative random variables. Let the conditional distribution of $X$ given a non-negative random variable $\theta$ be a Gumbel's bivariate exponential distribution with survival function

$$
\bar{F}\left(x_{1}, x_{2}\right)=e^{-\theta\left(x_{1}+x_{2}+x_{1} x_{2}\right)}, \quad x_{1}, x_{2}>0 .
$$

THEOREM. The distribution of $\theta$ is a mixture of two gamma distributions with respective parameters $\left(\alpha_{i}, \beta_{i}\right), i=1,2$ if and only if the unconditional distribution of $X$ is a mixture of two bivariate Pareto distributions (of the second type) with respective parameters $\left(\alpha_{i}, \beta_{i}\right), i=1,2$.

Proof: Suppose $\theta$ has a mixture distribution of two gamma distributions with density function

$$
\begin{gathered}
d G(\theta)=\sum_{i=1}^{2} \frac{\lambda_{i}}{\Gamma\left(\alpha_{i}\right)} \beta_{i}^{\alpha_{i}} \theta^{\alpha_{i}-1} e^{-\beta_{i} \theta} d \theta \\
\theta>0 ; \alpha_{i}>0 ; \beta_{i}>0 ; i=1,2 ; 0 \leqslant \lambda_{1} \leqslant 1 ; \lambda_{1}+\lambda_{2}=1 .
\end{gathered}
$$

Then the unconditional distribution function $F\left(x_{1}, x_{2}\right)$ of $X$ is given by

$$
\begin{aligned}
F\left(x_{1}, x_{2}\right) & =\int_{0}^{\infty} F\left(x_{1}, x_{2} \mid \theta\right) d G(\theta) \\
& =\sum_{i=1}^{2} \lambda_{i} \frac{\beta_{i}^{\alpha_{i}}}{\Gamma\left(\alpha_{i}\right)} \int_{0}^{\infty}\left[1-e^{-\theta x_{1}}-e^{-\theta x_{2}}+e^{-\theta\left(x_{1}+x_{2}+x_{1} x_{2}\right)}\right] \theta^{\alpha_{i}-1} e^{-\beta_{i} \theta} d \theta \\
& =\sum_{i=1}^{2} \lambda_{i}\left[1-\left(\frac{\beta_{i}}{\beta_{i}+x_{1}}\right)^{\alpha_{i}}-\left(\frac{\beta_{i}}{\beta_{i}+x_{2}}\right)^{\alpha_{i}}+\left(\frac{\beta_{i}}{\beta_{i}+x_{1}+x_{2}+x_{1} x_{2}}\right)^{\alpha_{i}}\right]
\end{aligned}
$$

The right hand side of (3.3) is the distribution function of the mixture of two bivariate Pareto distributions with respective parameters $\left(\alpha_{i}, \beta_{i}\right), i=1,2$, (see [5, Chapter 42,3]).

. Now, we prove the "only if" part.

Suppose that the unconditional distribution of $X$ is in the form of the right hand side of (3.3). Then we have

$$
\begin{aligned}
& \int_{0}^{\infty}\left[1-e^{-\theta x_{1}}-e^{-\theta x_{2}}+e^{-\theta\left(x_{1}+x_{2}+x_{1} x_{2}\right)}\right] d G(\theta) \\
& =\sum_{i=1}^{2} \lambda_{i}\left[1-\left(\frac{\beta_{i}}{\beta_{i}+x_{1}}\right)^{\alpha_{i}}-\left(\frac{\beta_{i}}{\beta_{i}+x_{2}}\right)^{\alpha_{i}}+\left(\frac{\beta_{i}}{\beta_{i}+x_{1}+x_{2}+x_{1} x_{2}}\right)^{\alpha_{i}}\right] .
\end{aligned}
$$


If $g(s)$ is the Laplace-Stieltjes transform of $G(\theta)$, then (3.4) may be written as

$$
\begin{aligned}
& g\left(x_{1}\right)+g\left(x_{2}\right)-g\left(x_{1}+x_{2}+x_{1} x_{2}\right) \\
& \quad \sum_{i=1}^{2} \lambda_{i}\left[\left(\frac{\beta_{i}}{\beta_{i}+x_{1}}\right)^{\alpha_{i}}+\left(\frac{\beta_{i}}{\beta_{i}+x_{2}}\right)^{\alpha_{i}}-\left(\frac{\beta_{i}}{\beta_{i}+x_{1}+x_{2}+x_{1} x_{2}}\right)^{\alpha_{i}}\right]
\end{aligned}
$$

or,

$$
W\left(x_{1}\right)+W\left(x_{2}\right)=W\left(x_{1}+x_{2}+x_{1} x_{2}\right)
$$

where,

$$
W(x)=g(x)-\sum_{i=1}^{2} \lambda_{i}\left(\frac{\beta_{i}}{\beta_{i}+x}\right)^{\alpha_{i}} .
$$

Now, since $W(0)=0$, then (3.5) is valid for $x_{1}=0$ (and valid, also, for $x_{2}=0$ ).

Therefore, following the argument of the proof of Theorem 2, p.41 in [1], it is easy to see that

$$
W(x)=0
$$

is the only solution of the functional equation (3.5). Hence,

$$
g(s)=\sum_{i=1}^{2} \lambda_{i}\left(\frac{\beta_{i}}{\beta_{i}+s}\right)^{\alpha_{i}} .
$$

The right hand side of (3.7) is the Laplace transform of the mixture of two gamma distributions with parameters $\left(\alpha_{i}, \beta_{i}\right), i=1,2$ respectively. Since the Lapalce transform is a one-to-one mapping the proof is complete.

REMARK. Suppose $F\left(x_{1}, x_{2} \mid \theta\right)$ is the distribution function of a bivariate Weibull distribution, that is

$$
\begin{gathered}
F\left(x_{1}, x_{2} \mid \theta\right)=1-e^{-\theta \gamma_{1} x_{1}^{b}}-e^{-\theta \gamma_{2} x_{2}^{b}}+e^{-\theta\left(\gamma_{1} x_{1}^{b}+\gamma_{2} x_{2}^{b}+r \gamma_{1} \gamma_{2} x_{1}^{b} x_{2}^{b}\right)}, \\
x_{1}, x_{2}>0 ; \gamma_{1} ; \gamma_{2}>0 ; b>0 ; r>0 .
\end{gathered}
$$

Then $\theta$ has a mixture distribution of two gamma distributions with respective parameters $\left(\alpha_{i}, \beta_{i}\right), i=1,2$ if and only if the unconditional distribution of $X$ is a mixture of two bivariate Burr distributions (see [3]) with respective parameters $\left(\alpha_{i}, \beta_{i}, r, \gamma_{1}, \gamma_{2}, b\right), i=1,2$.

In fact, the proof of this result is similar to that of the above theorem. The only difference is that, in the "only if" part of the proof we get the functional equation

$$
W\left(\gamma_{1} x_{1}^{b}\right)+W\left(\gamma_{2} x_{z}^{b}\right)=W\left(\gamma_{1} x_{1}^{b}+\gamma_{2} x_{2}^{b}+r \gamma_{1} \gamma_{2} x_{1}^{b} x_{2}^{b}\right)
$$

which, on substituting $y_{i}=\gamma_{i} x_{i}^{b}, i=1,2$, reduces to

$$
W\left(y_{1}\right)+W\left(y_{2}\right)=W\left(y_{1}+y_{2}+r y_{1} y_{2}\right)
$$

and this last equation has the same unique solution as that of equation (3.5). 


\section{ReFERENCES}

[1] J. Aczel, Lectures on functional equations and their applications (Academic Press, New York, 1966).

[2] W.D. Ashton, 'Distribution for gaps in road traffic', Inst. Maths. and Applics. 7 (1971), 37-46.

[3] F.C. Durling, Bivariate Probit, Logit and Burrit Analysis, THEMIS, Report No. 41 (Stat. Dept., Southern Methodist Univ., Dallas, Texas, 1969).

[4] J. Engel, M. Zijlstra and N.V. Philips, 'A characterization of the gamma distribution by the negative binomial distribution', J. Appl. Probab. 17 (1980), 1138-1144.

[5] N.L. Johnson and S. Kotz, Distributions in statistics 4 (Wiley, New York, 1972).

[6] A.W. Marshall and I. Olkin, 'A generalized bivariate exponential distribution', J. Appl. Probab. 4 (1967), 291-302.

[7] T.J. O'Neill and H.C. O'Neill, 'A gamma model for extra-binomial variation in dilution assays', Biometrics 49 (1993), 237-242.

[8] R.L. Prentice, 'Correlated binary regression with covariates specific to each binary observation', Biometrics 44 (1988), 1033-1048.

\footnotetext{
Mathematics Department

Faculty of Science

Ain Shams University

Cairo

Egypt
} 